Int. J. Electrochem. Sci., 15 (2020) 9579 - 9589

\title{
Ni-Mo Nanorod Bundles Grown within Nickel Foam for Excellent Electrochemical Performance
}

\author{
Jie Yuan ${ }^{1}$,Hui Zhao ${ }^{1}$, Ruizhuo Ouyang ${ }^{*}$ Yuqing Miao* \\ Bismuth Science Research Institute, University of Shanghai for Science and Technology, Shanghai \\ 200093, P. R. China \\ "E-mail: ouyangrz@usst.edu.cn (R. Ouyang); yqmiao@usst.edu.cn (Y. Miao). \\ ${ }^{1} \mathrm{~J}$. Yu and H. Zhao contributed equally to this work and should be considered as joint first authors
}

doi: $10.20964 / 2020.10 .08$

Received: 2 February 2020 / Accepted: 10 April 2020 / Published: 31 August 2020

\begin{abstract}
In this work, nanorod bundles of Ni-Mo-O were synthesized within nickel foam (NF) using a conventional hydrothermal method, which was the further vulcanized to form Ni-Mo-S upon doping sulfur. The NF/Ni-Mo-O and NF/Ni-Mo-S materials were characterized in terms of their morphology, composition and electrochemical properties. NF/Ni-Mo-O showed excellent performance as a supercapacitor. In addition, NF/Ni-Mo-S catalyzed the electrochemical oxidation of glucose in alkaline medium, exhibiting its potential application as a new fuel cell. Their excellent electrochemical performances greatly benefited from the preferred activity of NF/Ni-Mo-O and NF/Ni-Mo-S, efficient electron transfer over the electrode and high conductivity of NF.
\end{abstract}

\section{FULL TEXT}

(C) 2020 The Authors. Published by ESG (www.electrochemsci.org). This article is an open access article distributed under the terms and conditions of the Creative Commons Attribution license (http://creativecommons.org/licenses/by/4.0/). 